\title{
Abnormal Interhemispheric Connectivity in Neonates with D-Transposition of the Great Arteries Undergoing Cardiopulmonary Bypass Surgery
}

M. Makki, I. Scheer, C. Hagmann, R. Liamlahi, W. Knirsch, H. Dave, V. Bernet, K. Batinic, and B. Latal

\begin{abstract}
BACKGROUND AND PURPOSE: Neonates with severe CHD require CPB within the first days of life. White matter injury can occur before surgery, and this may impair the long-term neurodevelopmental and psychosocial outcome. The purpose of this study was to assess the microstructural development of the CC in infants with CHD before and after CPB for transposition of the great arteries.
\end{abstract}

MATERIALS AND METHODS: Fifteen patients with $\mathrm{CHD}$ and 11 age-matched $\mathrm{HC}$ were recruited. We separately quantified the parallel $\left(\mathrm{E}_{1}\right)$ and perpendicular $\left(E_{23}\right)$ diffusions, the $A D C$, and $F A$ of the genu of the $C C$ and splenium of the $C C$ before and after surgery.

RESULTS: In presurgical measures of the genu of the CC, higher $\mathrm{E}_{23}(P=.018)$, higher $\operatorname{ADC}(P=.026)$, and lower FA $(P=.033)$ values were measured compared with those in $\mathrm{HC}$. In the postsurgery scans, the genu of the $C C$ had higher $\mathrm{E}_{23}(P=.013)$, higher $\mathrm{ADC}(P=.012)$, and lower FA $(P=.033)$ values compared with those in HC. There was no significant difference in any DTI indices between the pre- and postsurgical groups.

CONCLUSIONS: We report abnormal microstructural development in the genu of the CC of infants with d-TGA before and after CPB. High $E_{23}$, high $A D C$, and low FA values in the genu of the $C C$ may be explained by abnormal axonal pruning, thinner myelin sheaths, smaller axonal diameters, or more oligodendrocytes. It appears that the genu of the CC is more vulnerable than the splenium of the CC in patients with CHD and may serve as a biomarker to identify infants at highest risk for adverse neurodevelopmental outcome.

ABBREVIATIONS: $\mathrm{CC}=$ corpus callosum; $\mathrm{CHD}=$ congenital heart disease; $\mathrm{CPB}=$ cardiopulmonary bypass surgery; $\mathrm{d}-\mathrm{TGA}=$ dextrotransposition of the great artery; $\mathrm{FA}=$ fractional anisotropy; $\mathrm{HC}=$ healthy controls

$\mathbf{T}$ he CC is the largest mass of the WM tracts that crosses the 2 cerebral hemispheres connecting bilaterally most of the cortical areas of the human brain. The primary role of the transcallosal fibers is to provide a functional and anatomic interhemispheric connection with defined areas containing fibers that serve cognitive, motor, speech, and vision functions. ${ }^{1-3}$ The basic structure of the CC is completed by 18-20 weeks' gestation but continues to increase in size during the third trimester ${ }^{4}$ and grows dramatically from anterior to posterior during the first 2 postnatal years, ${ }^{5}$ while preoligodendrocytes are thought to appear at the extremities (eg, the genu and splenium). ${ }^{6}$ Previous studies re-

Received March 29, 2012; accepted after revision May 30.

From the Department of Diagnostic Imaging (M.M., I.S.), Division of Cardiology (R.L., W.K., K.B.), Division of Congenital Cardiovascular Surgery (H.D.), Pediatric Intensive Care and Neonatology (V.B.), and Child Development Center (B.L.), University Children's Hospital, Zurich, Switzerland; and Clinic of Neonatology (C.H.), University Hospital of Zurich, Zurich, Switzerland.

Please address correspondence to Malek Makki, PhD, University Children's Hospital, Magnetic Resonance Center, Steinwiesstr 75, 8032 Zurich, Switzerland; e-mail: malek.makki@kispi.uzh.ch

Evidence-Based Medicine Level 2.

http://dx.doi.org/10.3174/ajnr.A3256 ported that the morphology of the CC is influenced by cortical asymmetry, ${ }^{7}$ forebrain volume, ${ }^{8}$ and functional lateralization. ${ }^{9}$ Patients with severe CHD require cardiopulmonary bypass surgery within the neonatal period. However, long-term neurodevelopmental and psychosocial outcome may be impaired. ${ }^{10,11}$

White matter injury and delayed intrauterine brain maturation may also play an important role in an adverse outcome. ${ }^{12,13}$ More than half of neonates with severe forms of CHD already manifest neurobehavioral and neurologic abnormalities such as muscular hypotonia or poor feeding before surgery. ${ }^{14}$ Motor deficits may occur in up to $40 \%$ of children after CPB, and intellectual and neuromotor impairments might manifest in adolescence. ${ }^{15}$ Recent MR imaging studies showed that in more than one-third of neonates with CHD, brain injury was detected already preoperatively; and in one-third of the neonates, acquired brain injuries occurred during or shortly after cardiac surgery. ${ }^{16,12}$ However, little is known of the characterization and development of the CC around the time of birth for full-term neonates with $\mathrm{CHD}$ in relation to surgical procedures.

DTI is an MR imaging technique used to characterize the microstructure of large WM tracts in physiologic and pathologic 
Table 1: Characteristics of the 15 patients who had both pre- and postoperative MRI, including DTI, and 11 healthy controls

\begin{tabular}{|c|c|c|c|c|c|c|c|c|c|c|}
\hline & \multicolumn{5}{|c|}{ Patients $(n=15)$} & \multicolumn{5}{|c|}{ Healthy Controls $(n=11)$} \\
\hline & Mean & SD & Min & Max & Med & Mean & SD & Min & Max & Med \\
\hline Gestational age (wk) & 39 & 1 & 37 & 41 & 39 & 39 & 1 & 38 & 41 & 40 \\
\hline Birth weight (g) & 3381 & 452 & 2560 & 3990 & 3340 & 3466 & 417 & 3000 & 4160 & 3500 \\
\hline From birth to 1st MRI (days) & 8 & 6 & 3 & 27 & 8 & 26 & 5 & 15 & 32 & 26 \\
\hline From birth to 2nd MRI (days) & 29 & 9 & 16 & 52 & 27 & & & & & \\
\hline From lst to 2nd MRI (days) & 21 & 8 & 11 & 42 & 20 & & & & & \\
\hline From surgery to 2nd MRI (days) & 14 & 6 & 7 & 28 & 12 & & & & & \\
\hline
\end{tabular}

Note:-Min indicates minimum; Max, maximum; Med, median.

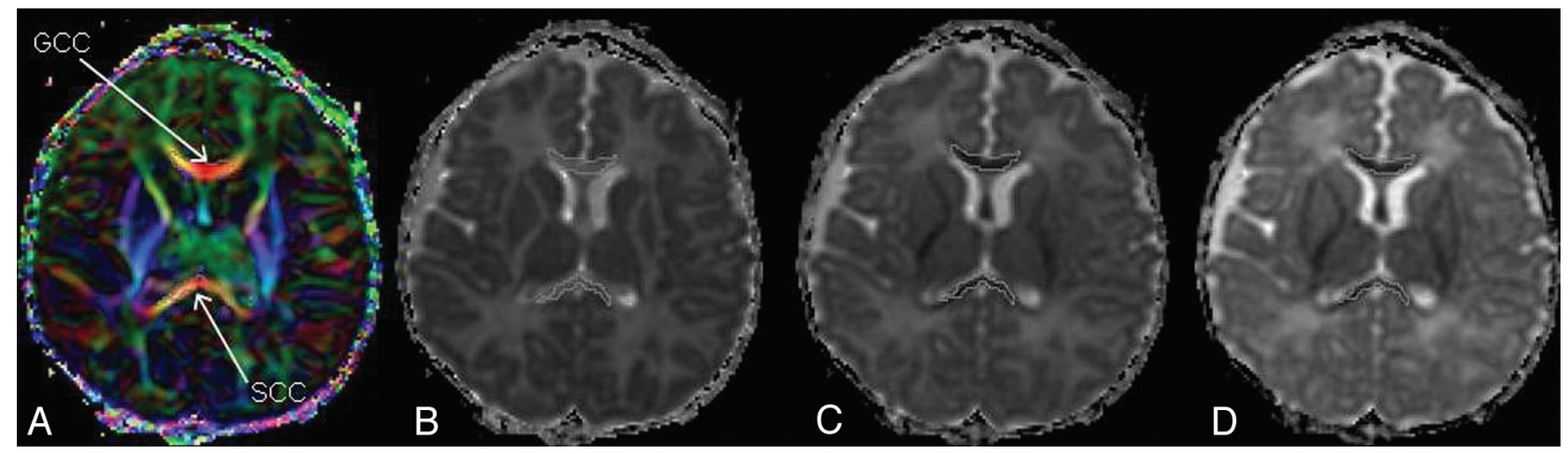

FIG 1. Direction-encoded color map $(A)$ showing the genu of the $C C$ and splenium of the $C C$ in red, meaning that the fibers are left-right oriented (or vice versa). The ROIs are reproduced on the main eigenvalue, $E_{1}(B)$; the middle eigenvalue, $E_{2}(C)$; and the minor eigenvalue, $E_{3}$, maps $(D)$.

conditions and provides complex measures that reflect various microstructural properties of the WM, including axon diameter, myelination, and fiber attenuation. ${ }^{17}$ Microstructural changes in the CC have been described with DTI studies in premature infants, ${ }^{18}$ extremely preterm infants, ${ }^{19}$ very-low-birth-weight adolescents, ${ }^{20}$ and preterm born young adults. ${ }^{21}$

The overall aims of this study were to prospectively investigate WM microstructural development in the interhemispheric brain connectivity in neonates with d-TGA before and after CPB. We also aimed to assess WM growth of neonates with d-TGA by comparing it with that in age-matched healthy controls. We hypothesized that neonates with CHD would have delayed myelin maturation compared with age-matched healthy controls in early myelinated WM tracts such as the splenium and genu of the CC.

\section{MATERIALS AND METHODS \\ Subjects}

Twenty-eight neonates with confirmed d-TGA were prospectively enrolled between December 2009 and October 2011. Fifteen patients (10 males) consecutively underwent both pre- and postoperative successful DTI examinations. Mean postconceptional age was 39 weeks (ranging from 37 to 41 weeks). Preoperative MR imaging was performed on average $8 \pm 6$ days after birth. The postsurgical MR imaging was performed on average 14 days after $\mathrm{CPB}$ (ranging from 7 to 28 days) and $21 \pm 8$ days following the first MR imaging (Table 1). Eleven healthy term neonates (5 males) were recruited and were examined with the same DTI sequence to serve as HC (Table 1). The institutional ethics committee approved this study, and written informed consent was obtained from guardians.

\section{MR Imaging Acquisition and Analysis}

The MR imaging studies were performed on a 3T scanner by using the following: 1) volumetric T1WI with a 3D magnetization-prepared spoiled gradient-echo sequence $(\mathrm{TR} / \mathrm{TE} / \mathrm{TI}=7.9 / 3.0 / 450 \mathrm{~ms}$, flip angle $=20^{\circ}, \mathrm{FOV}=25.6 \mathrm{~cm}^{2}$, matrix $=256 \times 256$, parallel imaging, thickness $=2 /-1 \mathrm{~mm}$ gap);2) T2-weighted fast spin-echo series $(\mathrm{NEX}=2$, thickness $=2.5 / 0.2 \mathrm{~mm}$ gap, $\mathrm{FOV}=18 \mathrm{~cm} \times$ $75 \%)$, matrix $=512 \times 320$ for the axial plane and $384 \times 320$ for the coronal plane; 3) 2 single-voxel MR spectroscopy examinations, 1 in the basal ganglia and 1 in the parietal WM $(\mathrm{TR} / \mathrm{TE}=3000 / 35 \mathrm{~ms}$, NEX $=8$, thickness $=17 \mathrm{~mm}^{2}$ ); and 4) DTI consisting of double refocusing pulses and 1 image volume with no diffusion weighting $\left(b=0 \mathrm{~s} / \mathrm{mm}^{2}\right)$, followed by 35 gradient-direction acquisitions $\left(b=700 \mathrm{~s} / \mathrm{mm}^{2}\right)$. The matrix was $128 \times 128$ (reconstructed to $256 \times$ 256), $\mathrm{FOV}=22 \mathrm{~cm}^{2}$, and TR/TE $=9000 / 90 \mathrm{~ms}$.

The genu of the CC and splenium of the CC were chosen because they represent, respectively, the anterior and posterior parts of the interhemispheric connectivity. Two observers processed the images; 1 was blinded to the acquisition and repeated the processing to compare inter- and intraobserver variability. The Pearson test was used to check the correlation of the 3 measures. Polygonal regions of interest were manually drawn at the edge of each structure on the directionally color-encoded maps (Fig 1), and care was taken to avoid partial voluming effects. Each region of interest was automatically copied and pasted on the $\mathrm{E}_{1}$, $\mathrm{E}_{2}$, and $\mathrm{E}_{3}$ maps. Previous DTI evidence demonstrated that derived parallel and perpendicular diffusivities can detect and separate axonal from myelin damage ${ }^{22-24}$; thus, we separately measured the 3 eigenvalues, $E_{1}, E_{2}$, and $E_{3}$. Subsequently, values of parallel diffusion $\left(E_{1}\right)$, perpendicular diffusion $\left[E_{23}=\left(E_{1}+E_{2}\right)\right.$ 12], ADC, and FA were then calculated on each region of interest. 
Table 2: Percentage changes corrected for age in the GCC and SCC measured among Post, Pre, and HC groups

\begin{tabular}{|c|c|c|c|c|c|c|c|c|}
\hline & \multicolumn{4}{|c|}{ GCC } & \multicolumn{4}{|c|}{ SCC } \\
\hline & $\left.\mathrm{E}_{1} \%\right)$ & $E_{23}(\%)$ & ADC (\%) & FA (\%) & $\mathrm{E}_{1}(\%)$ & $E_{23}(\%)$ & ADC (\%) & FA (\%) \\
\hline Pre vs HC & 1.68 & 10.94 & 5.79 & -8.20 & -0.84 & 13.93 & 5.30 & -10.48 \\
\hline Post vs HC & 4.93 & 17.54 & 10.35 & -9.40 & 0.60 & 13.98 & 6.18 & -9.58 \\
\hline Pre vs Post & 2.35 & 8.79 & 5.28 & -6.33 & -1.03 & 5.69 & 1.94 & -6.26 \\
\hline
\end{tabular}

Note:-Pre indicates presurgery; Post, postsurgery; GCC, genu of the CC; SCC, splenium of the CC.
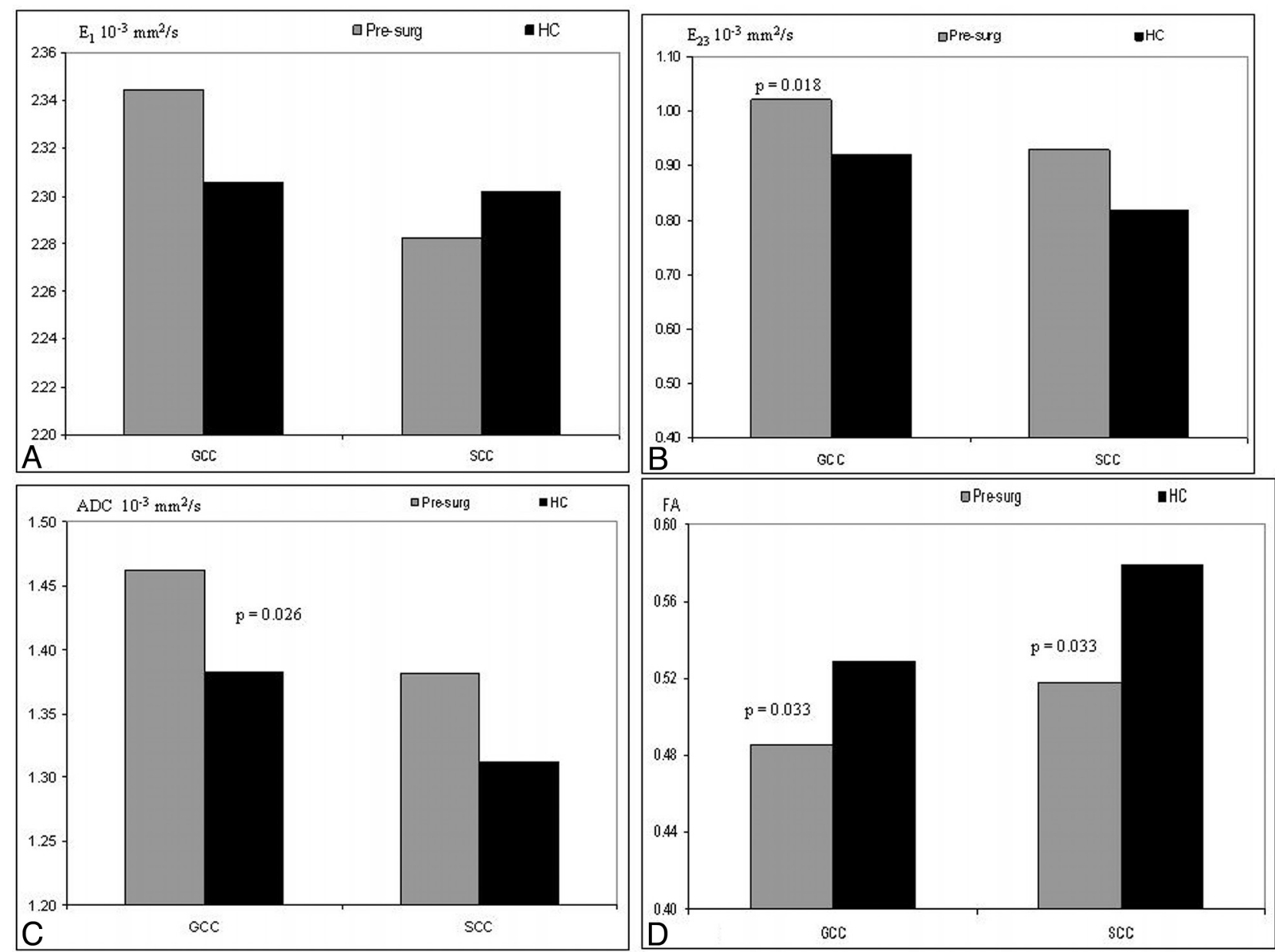

FIG 2. Estimated marginal means corrected for postconceptional age of parallel diffusion (A), perpendicular diffusion (B), apparent diffusion coefficient $(C)$, and fractional anisotropy $(D)$ of the $\mathrm{HC}$ and presurgical (presurg) groups. The DTI of the genu of the $C C$ in the presurgical patients indicates a significantly higher $\mathrm{E}_{23}(P=.018)$, higher $\mathrm{ADC}(P=.026)$, and lower FA $(P=.033)$. In the splenium of the CC, we measured significantly lower FA $(P=.033)$ in the presurg groups compared with the $\mathrm{HC}$.

The between-group statistical comparison (presurgery, postsurgery, healthy controls) was performed separately for each structure by using a general linear model multiple analysis of covariance (Statistical Package for the Social Sciences, Version 14.0; SPSS, Chicago, Illinois). Group differences with $P<.05$ were considered significant. Postconceptional age at MR imaging of the subjects was included as a covariate because of age-related increases in FA and decreases in ADC. ${ }^{25,26}$

\section{RESULTS}

Intra- and interobserver correlations of $\mathrm{E}_{1}$ were, respectively, 0.947 and 0.915 for the genu of the CC and 0.801 and 0.867 for splenium of the CC. MR spectroscopy findings were abnormal in all patients, with elevated lactate in the WM and basal ganglia and decreased $N$-acetylaspartate values.
In the genu of the $\mathrm{CC}$, comparison between the presurgery $\mathrm{CHD}$ group and the $\mathrm{HC}$ group revealed higher values in $\mathrm{E}_{1}$ and $\mathrm{E}_{23}$, leading to higher $\mathrm{ADC}$ values and lower $\mathrm{FA}$ values for the CHD group. Postsurgical DTI indices of patients with CHD compared with $\mathrm{HC}$ showed a similar pattern with increased values in $\mathrm{E}_{1}, \mathrm{E}_{23}$, and $\mathrm{ADC}$ and a decrease in FA compared with HC. Although there were approximately 21 days between the pre- and postsurgical MR imaging in the CHD group, differences between pre- and postsurgery DTI measures showed a similar trend with an increase in $\mathrm{E}_{1}, \mathrm{E}_{23}$, and $\mathrm{ADC}$ and a decrease in FA with time.

In the splenium of the CC, preoperative age-corrected differences in the DTI metrics showed increased values in both $\mathrm{E}_{23}$ (13.93\%) and $\operatorname{ADC~(5.3\% )~and~a~decreased~value~in~} \mathrm{FA}$ 

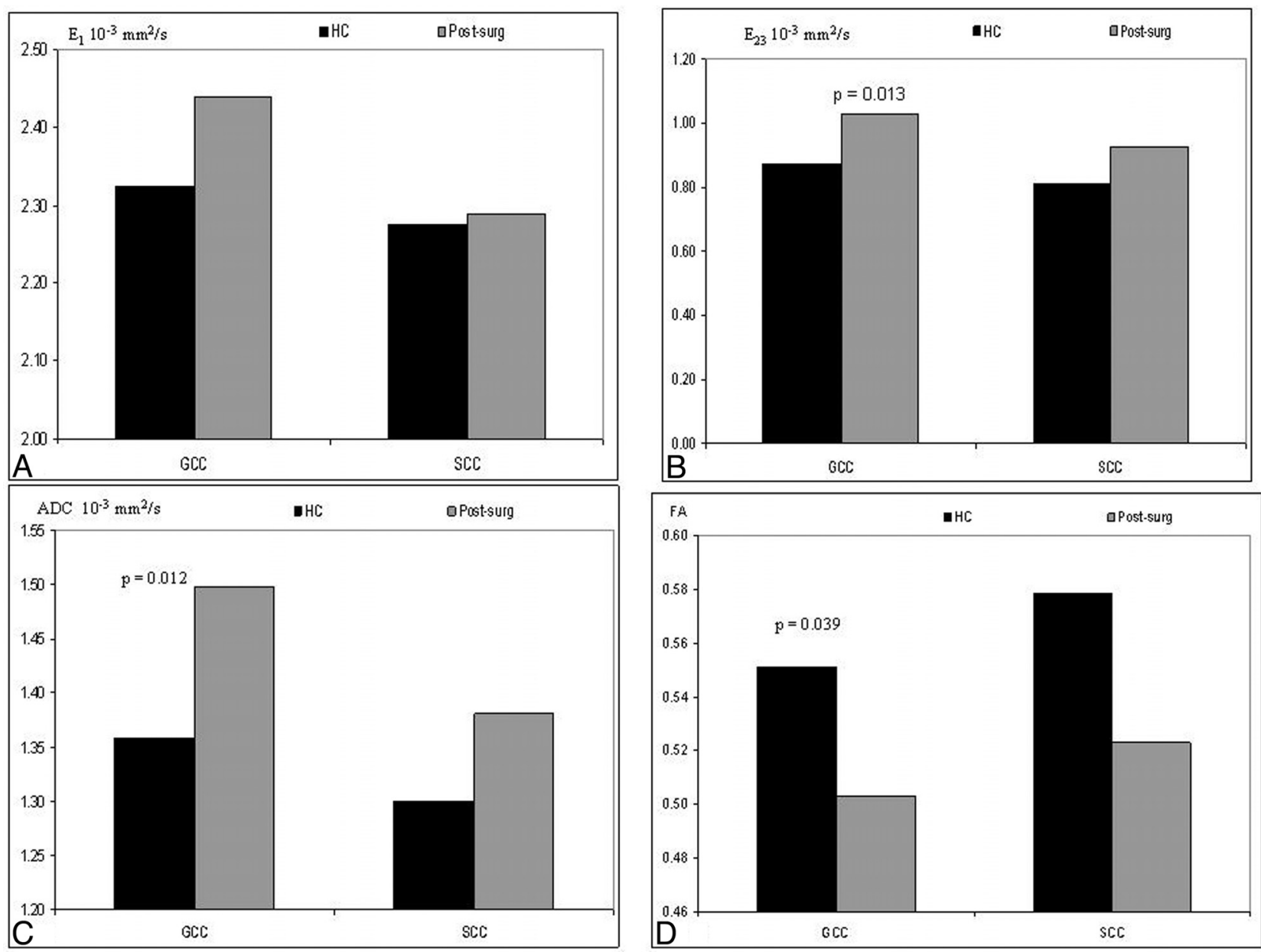

FIG 3. Estimated marginal means corrected for postconceptional age of parallel diffusion, $E_{1}(A)$; perpendicular diffusion, $E_{23}(B)$; $A D C(C)$; and $F A$ $(D)$ of the $\mathrm{HC}$ and postsurgical (postsurg) groups. The DTI in the genu of the CC in the postsurg group indicated a significantly higher $E_{23}(P=.018)$, higher ADC $(P=.026)$, and lower FA $(P=.033)$ than those in the HC group. No differences were reported in the splenium of the CC.

$(-10.48 \%)$ compared with the HC group (Table 2). Similarly, we measured positive differences between the postsurgery and $\mathrm{HC}$ groups (corrected for age) in $\mathrm{E}_{23}(13.98 \%)$ and $\mathrm{ADC}(6.18 \%)$ and negative difference in FA $(-9.58 \%)$. We also reported increases in both $\mathrm{E}_{23}(5.69 \%)$ and $\mathrm{ADC}(1.94 \%)$ and a decrease in FA $(-6.26 \%)$ between the presurgical and postsurgical examinations (Table 2). General linear model analysis controlling for gestational age revealed significant differences in $\mathrm{E}_{23}$ (genu of the CC: $P=.01$; splenium of the CC: $P=.026$ ), ADC (genu of the CC: $P$ $=.002$; splenium of the CC: $P=.05$ ), and FA (genu of the CC: $P$ $=.006$; splenium of the CC: $P=.021)$.

Presurgery versus HC. Post hoc analysis between the $\mathrm{HC}$ and the presurgery CHD group measures in the genu (Fig 2) demonstrated significantly lower $\mathrm{E}_{23}(P=.018)$, higher $\operatorname{ADC}(P=.026)$, and lower FA $(P=.033)$. There was no significant difference in the parallel diffusion between these 2 groups. In the splenium of the CC, the presurgery group had a significantly lower FA ( $P=$ .033 ) compared with HC, and all other indices were not significantly different.

Postsurgery versus HC. With regard to the postsurgery group measures, the post hoc analysis in the genu of the CC indicated a significantly lower $\mathrm{E}_{23}(P=.013)$, higher $\operatorname{ADC}(P=.012)$, and lower FA ( $P=.039)$ compared with the HC group (Fig 3$)$. In the splenium of the CC, we did not measure any significant difference between these 2 groups.

Postsurgery versus Presurgery. We observed an abnormal increase in both $\mathrm{E}_{23}$ and $\mathrm{ADC}$ with a simultaneous decrease in $\mathrm{FA}$ in both the genu of the CC and splenium of the CC (Fig 4). With regard to $\mathrm{E}_{1}$, there was a nonsignificant increase in the genu of the CC and a nonsignificant decrease in the splenium of the CC.

\section{DISCUSSION}

The main findings were delayed WM maturation in the genu of the CC of neonates with d-TGA both before and after surgery compared with healthy control neonates. In the genu of the CC of the presurgical examinations, we measured higher $\mathrm{E}_{23}$ and $\mathrm{ADC}$ and lower FA compared with the HC group. Similarly, the postsurgery patients demonstrated higher $\mathrm{E}_{23}$ and $\mathrm{ADC}$ and lower FA compared with the HC group. Furthermore, the genu of the CC was more affected with higher $\mathrm{E}_{23}$ and $\mathrm{ADC}$ compared with the splenium of the CC in both the presurgical and postsurgical measures. We also observed that between pre- and postsurgical measures of these neonates, there was an abnormal increase in both $\mathrm{E}_{23}$ and $\mathrm{ADC}$, with a simultaneous nonsignificant decrease in FA 

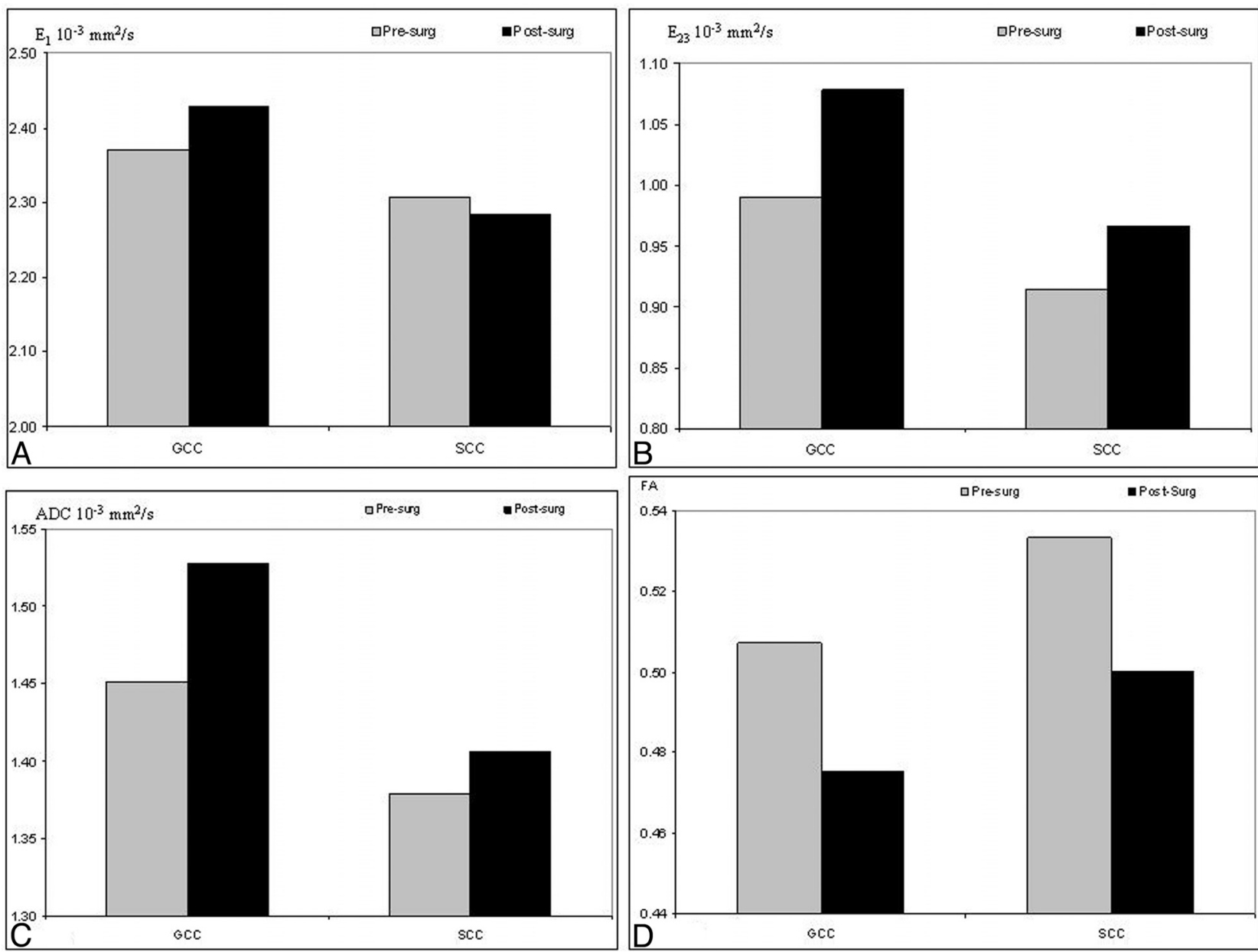

FIG 4. Estimated marginal means corrected for postconceptional age of parallel diffusion, $E_{1}(A)$; perpendicular diffusion, $E_{23}(B)$; $A D C(C)$; and $F A$ $(D)$ of the postsurgical (postsurg) and presurgical (presurg) groups. DTI in the genu of the CC and splenium of the CC in the postsurg group reveals higher $\mathrm{E}_{23}$, higher ADC, and lower FA than those in the presurg group. The $\mathrm{E}_{1}$ is higher in the genu of the CC and lower in the splenium of the CC of the postsurg compared with the presurg group, but these differences are significant.

in both structures of the CC. However, one would expect an opposite pattern of change in the normally developing brain.

Our findings are clinically important and support the hypothesis that neonates with severe CHD already have delayed brain development before surgery. ${ }^{12,13}$ Most important, we could show that the genu of the CC is particularly affected or may serve as a marker for this maturational delay. Furthermore, instead of a progression of maturation with a decrease in ADC and increase in FA values, the period around the surgery was associated with a reverse development. It is not clear whether this is a transient process without clinical relevance. The neurodevelopmental profiles in such neonates are similar to those in premature neonates and may play an important role in planning an in utero cardiac intervention. However, our postsurgical findings suggest that brain substrate is more important in long-term outcomes than surgical intervention techniques.

Recent DTI studies in the CC of infants ${ }^{27}$ and adults ${ }^{28}$ showed that $\mathrm{FA}, \mathrm{ADC}, \mathrm{E}_{1}$, and $\mathrm{E}_{23}$ varied among regions. Anisotropy was highest in the splenium of the CC followed by the genu of the CC and lowest in the callosal body. The ADC values showed the opposite pattern, being highest in the body, followed by the splenium of the CC, and lowest in the genu of the CC. Aboitiz et $\mathrm{al}^{29}$ demonstrated an increase in the absolute fiber attenuation from the genu of the CC to the splenium of the CC and a decrease toward the posterior midbrain and in the most posterior part of the splenium. It has been shown that in healthy subjects, the variation in FA was correlated with axon attenuation, diameter, demyelination, or less densely packed thin fibers. ${ }^{30}$ The observed decreases in FA that were accompanied by increased $\mathrm{E}_{23}$ in patients with d-TGA compared with HC support the hypothesis that differences in myelination account for those changes, which might be related to the microstructural organizational differences of the anterior CC. ${ }^{31}$ The amplified decreases in FA detected in this genu of the CC indicate a disturbance of developing fibers that project through these WM areas and may arise from the severe astrocyte hypertrophy ${ }^{23}$ detected in this structure in these patients. Astrocyte hypertrophy and oligodendrocyte death accompanying the myelin deficit have been shown to play a crucial role in the amplification of water diffusion coefficients in animal models because cytoplasm extensions of astrocytes follow the axonal pathway longitudinally. ${ }^{24}$

In the splenium of the CC, there was a lower FA value in the presurgery patients than the HC group. However, no significant difference was detected between the HC group and the postsur- 
gery MR imaging, suggestive of either brain plasticity or fast remyelination of the fibers. Further investigations are required to determine whether these changes are the consequences of the surgical procedure or normal brain development. With regard of parallel diffusion $\left(\mathrm{E}_{1}\right)$, our study also showed a mild nonsignificant increase in patients compared with $\mathrm{HC}$, which might be the consequence of neurofibril destruction, ${ }^{31}$ fiber coherence, ${ }^{32}$ and axonal injury. ${ }^{33}$ Therefore, it is likely that changes in parallel diffusivity reflect complex interactions of multiple biologic factors that drive it in different directions. In addition, we found disruption of the microstructural organization of WM in the genu as reflected by higher $\mathrm{E}_{23}$ in patients with d-TGA than in HC. Reduction of $\mathrm{E}_{23}$ during WM maturation was attributed to myelination because this modulates the diffusion anisotropy originating from cell membranes by creating an additional barrier. ${ }^{17}$ Modifications of the intracellular or extracellular spaces and the abnormalities contributed by astrocytic activation may influence the perpendicular component of water diffusivity. The disruption of the myelin sheath has been found to increase perpendicular diffusion without affecting parallel diffusion. Furthermore, we observed an increase in both $\mathrm{ADC}$ and $\mathrm{E}_{23}$ in the genu of the $\mathrm{CC}$ of patients with d-TGA, both before and after surgery, suggesting that the anterior callosal WM is less mature in these patients than in HC neonates. The lack of microstructural integrity of the anterior interhemispheric connectivity in neonates with CHD is most likely due to impaired or delayed myelination or premyelination. The effects of WM prematurity within the CC have been studied with DTI in adolescents and infants. ${ }^{21,34}$ Very preterm infants had reduced anisotropy and increased $\mathrm{ADC}, \mathrm{E}_{1}$, and $\mathrm{E}_{23}$ in the posterior interhemispheric tracts. ${ }^{23}$

Considering that in the $\mathrm{CC}$ there is a posterior-to-anterior gradient of myelination, the late developing anterior regions may be more vulnerable to growth deficits in neonates with d-TGA. The microstructural development of the splenium, the early myelinating part of the CC, seems normally developed before and after surgery. Absolute fiber attenuation decreases from the genu to the posterior midbody of the CC, increases toward the splenium, and is reduced again in the most posterior part of the splenium. ${ }^{31}$ Within the CC, the clearest diffusion changes seen in the genu were increases of $\mathrm{ADC}$ and $\mathrm{E}_{23}$, indicating less mature WM and possibly delayed or disrupted myelination of the WM connecting the hemispheres of the prefrontal cortex involved in planning and cognition. It also demonstrates that the insult in the CC was more pronounced in the late myelinated region, and this suggests that it could start later in pregnancy rather than at an early stage (18 weeks' gestational age).

\section{Limitations}

The first limitation of our study is the rather small sample size (15 patients and 11 healthy controls). Recruiting and scanning these neonates in natural sleep before and after CPB are complicated procedures that require combined methodologic effort from the radiologist, cardiologist, surgeon, and neonatologist. The MR imaging protocol was optimized and shortened for the comfort of these extremely sick neonates; nevertheless, quite often they failed to finish either the pre- or postsurgical DTI, resulting in a reduced sample size. The second main issue was the image artifacts and geometric distortion, which are more pronounced around the cortical areas compared with midbrain at the level of the CC; thus, we processed the raw data.

\section{CONCLUSIONS}

There is evidence of abnormal microstructural development in the genu of the CC of infants before and after surgery for transposition of the great artery. Disrupted development of the genu may have implications for other frontal structures that develop in association with the anterior fibers. It is likely that there are fewer oligodendrocytes in the CC of patients with $\mathrm{CHD}$, which would explain the increased $\mathrm{ADC}$ and $\mathrm{E}_{23}$, which occur simultaneously with decreased FA. Together, all these differences in the genu of the CC rather than the splenium of the CC of neonates with $\mathrm{CHD}$ indicate that WM microstructure is more immature than that of $\mathrm{HC}$ neonates, and it is likely an ongoing process started at the fetal level and seems not affected by the surgical procedure. The lack of a significant difference in $E_{1}$ in the presence of $E_{23}$ alterations between $\mathrm{HC}$ neonates and patients with $\mathrm{CHD}$ would imply that the reduced microstructural integrity evident in preand postsurgical DTI is most likely due to impaired or delayed myelination or premyelination. These early changes in the genu of the CC may lead to higher rates of cognitive and motor impairment in infants with CHD.

Disclosure: Rabia Liamlahi—RELATED: Grant: Anna Mueller-Grocholski Foundation. * Walter Knirsch—RELATED: Grant: Anna Mueller-Grocholski Foundation, ${ }^{\star}$ Comments: The project was supported by the Anna Müller-Grocholski Foundation. Hitendu Dave-UNRELATED: Travel/Accommodations/Meeting Expenses Unrelated to Activities Listed: No money paid, but sponsorship for attendance at international meetings provided by Medtronic, Terumo, and so forth. No sponsorship to meetings was received for activities related to the submitted work. *Money paid to the institution.

\section{REFERENCES}

1. Caillé S, Sauerwein HC, Schiavetto A, et al. Sensory and motor interhemispheric integration after section of different portions of the anterior corpus callosum in nonepileptic patients. Neurosurgery 2005;57:50-59

2. Narberhaus A, Segarra D, Caldú X, et al. Corpus callosum and prefrontal functions in adolescents with history of very preterm birth. Neuropsychologia 2008;46:111-16

3. Kumar R, Macey PM, Woo MA, et al. Selectively diminished corpus callosum fibers in congenital central hypoventilation syndrome. Neuroscience 2011;178:261-69

4. Malinger G, Zakut $H$. The corpus-callosum: normal fetal development as shown by transvaginal sonography. AJR Am J Roentgenol 1993;161:1041-43

5. Richards LJ, Plachez C, Ren T. Mechanisms regulating the development of the corpus callosum and its agenesis in mouse and human. Clin Genet 2004;66:276-89

6. Hüppi PS, Murphy B, Maier SE, et al. Microstructural brain development after perinatal cerebral white matter injury assessed by diffusion tensor magnetic resonance imaging. Pediatrics 2001;107: 455-60

7. Dorion AA, Chantôme M, Hasboun D, et al. Hemispheric asymmetry and corpus callosum morphometry: a magnetic resonance imaging study. Neurosci Res 2000;36:9-13

8. Jäncke L, Staiger JF, Schlaug G, et al. The relationship between corpus callosum size and forebrain volume. Cereb Cortex 1997;7:48-56

9. Josse G, Seghier ML, Kherif F, et al. Explaining function with anatomy: language lateralization and corpus callosum size. $\mathrm{J} \mathrm{Neu}$ rosci 2008;28:14132-39

10. Gessler P, Schmitt B, Prètre R, et al. Inflammatory response and 
neurodevelopmental outcome after open-heart surgery in children. Pediatr Cardiol 2009;30:301-05

11. Majnemer A, Limperopoulos C, Shevell M, et al. Long-term neuromotor outcome at school entry of infants with congenital heart defects requiring open-heart surgery. J Pediatr 2006;148:72-77

12. Miller SP, McQuillen PS, Hamrick S, et al. Abnormal brain development in newborns with congenital heart disease. N Engl J Med 2007; 357:1928-38

13. Licht DJ, Shera DM, Clancy RR, et al. Brain maturation is delayed in infants with complex congenital heart defects. J Thorac Cardiovasc Surg 2009;137:529-36

14. Limperopoulos C, Majnemer A, Shevell MI, et al. Neurodevelopmental status of newborns and infants with congenital heart defects before and after open heart surgery. J Pediatr 2000;137:638-45

15. von Rhein M, Dimitropoulos A, Valsangiacomo-Buechel ER, et al. Risk factors for neurodevelopmental impairments in school-age children after cardiac surgery with full-flow cardiopulmonary bypass. J Thorac Cardiovasc Surg 2012;144:577-83

16. Mahle WT, Tavani F, Zimmerman RA, et al. An MRI study of neurological injury before and after congenital heart surgery. Circulation 2002;106:I109-14

17. Beaulieu C. The basis of anisotropic water diffusion in the nervous system: a technical review. NMR Biomed 2002;15:435-55

18. Hasegawa T, Yamada K, Morimoto M, et al. Development of corpus callosum in preterm infants is affected by the prematurity: in vivo assessment of diffusion tensor imaging at term-equivalent age. $P e$ diatr Res 2011;69:249-54

19. Skiöld B, Vollmer B, Böhm B, et al. Neonatal magnetic resonance imaging and outcome at age $\mathbf{3 0}$ months in extremely preterm infants. J Pediatr 2012;160:559-566.e1

20. Eikenes L, Lohaugen GC, Brubakk AM, et al. Young adults born preterm with very-low birth weight demonstrate widespread white matter alterations on brain DTI. Neuroimage 2011;54:1774-85

21. Thompson DK, Inder TE, Faggian N, et al. Characterization of the corpus callosum in very preterm and full-term infants utilizing MRI. Neuroimage 2011;55:479-90

22. Counsell SJ, Shen Y, Boardman JP, et al. Axial and radial diffusivity in preterm infants who have diffuse white matter changes on magnetic resonance imaging at term-equivalent age. Pediatrics 2006; $117: 376-86$
23. Harsan LA, Poulet P, Guignard B, et al. Astrocytic hypertrophy in dysmyelination influences the diffusion anisotropy of white matter. J Neurosci Res 2007;85:935-44

24. Drobyshevsky A, Song SK, Gamkrelidze G, et al. Developmental changes in diffusion anisotropy coincide with immature oligodendrocyte progression and maturation of compound action potential. J Neurosci 2005;25:5988-97

25. Hüppi PS, Maier SE, Peled S, et al. Microstructural development of human newborn cerebral white matter assessed in vivo by diffusion tensor magnetic resonance imaging. Pediatr Res 1998; 44:584-90

26. Schneider JF, Il'yasov KA, Hennig J, et al. Fast quantitative diffusiontensor imaging of cerebral white matter from neonatal period to adolescence. Neuroradiology 2004;46:258-66

27. Gao W, Lin W, Chen Y, et al. Temporal and spatial development of axonal maturation and myelination of white matter in the developing brain. AJNR Am J Neuroradiol 2009;30:290-96

28. Hofer S, Frahm J. Topography of the human corpus callosum revisited-comprehensive fiber tractography using diffusion tensor magnetic resonance imaging. Neuroimage 2006;32:989-94

29. Aboitiz F, Scheibel AB, Fisher RS, et al. Fiber composition of the human corpus callosum. Brain Res 1992;598:143-53

30. Wahl M, Lauterbach-Soon B, Hattingen E, et al. Human motor corpus callosum: topography, somatotopy, and link between microstructure and function. J Neurosci 2007;27:12132-38

31. Song SK, Yoshino J, Le TQ, et al. Demyelination increases radial diffusivity in corpus callosum of mouse brain. Neuroimage 2005;26: $132-40$

32. Dubois J, Dehaene-Lambertz G, Perrin M, et al. Asynchrony of the early maturation of white matter bundles in healthy infants: quantitative landmarks revealed noninvasively by diffusion tensor imaging. Hum Brain Mapp 2008;29:14-27

33. Kim JH, Budde MD, Liang HF, et al. Detecting axon damage in spinal cord from a mouse model of multiple sclerosis. Neurobiol Dis 2006;21:626-32

34. Constable RT, Ment LR, Vohr BR, et al. Prematurely born children demonstrate white matter microstructural differences at 12 years of age, relative to term control subjects: an investigation of group and gender effects. Pediatrics 2008;121:306-16 